\title{
Die Marketing-Expertin
}

KERSTIN WEIHS Homepage, Flyer, Annoncen - es gibt zahlreiche Möglichkeiten, seine Praxis nach außen hin zu präsentieren. Doch wie wirkt diese Werbung eigentlich auf den Patienten? Das fragte sich auch Kerstin Weihs und machte es zum Thema ihrer Bachelorarbeit. Ein Ergebnis ihrer Untersuchung: Die Homepage spielt in der Stadt eine größere Rolle als auf dem Land.

Inwieweit können Sie Ihre Expertise zur Werbung an Ihrem Arbeitsplatz einbringen?

Der Inhaber unseres Physiotherapiezentrums ist sehr offen für meine Anregungen. Seit er die Ergebnisse meiner Bachelorarbeit kennt, achtet er auf eine regelmäßige Aktualisierung der Inhalte auf unserer Homepage. Aufgrund der ländlichen Lage hatte die Mund-zu-Mund-Propaganda bisher den größten Stellenwert in der Werbung. Gerade die jüngere Generation findet uns aber zunehmend über das Internet.

Das heißt, die Investition in eine gute PraxisHomepage lohnt sich für Physiotherapeuten? Meiner Erfahrung nach ja. Unsere Patienten berichten, dass sie uns gut im Internet finden, keine Schwierigkeiten haben, sich auf unserer Homepage zurechtzufinden, und die Aktualität der Inhalte schätzen. Zudem beobachte ich, dass das Einzugsgebiet des Zentrums langsam größer wird. In welchem Maße das mit der Homepage zusammenhängt, kann ich natürlich nicht sagen. Aber sie ist sicherlich kein unwichtiger Faktor. 


\section{Kundenreaktion auf Werbemittel von Praxen}

\section{Die Bachelorarbeit}

Werbung ist in unserem Alltag stets präsent. Auch für Physiotherapieeinrichtungen ist sie ein gutes Mittel, um (potenzielle) Patienten auf sich aufmerksam zu machen beziehungsweise langfristig in Kontakt zu bleiben. Bisher reagieren die Inhaber aber oftmals zurückhaltend, denn viele sind unsicher, inwieweit Werbung im Heilmittelbereich überhaupt erlaubt ist. Als Dienstleister im Gesundheitswesen müssen sich Physiotherapeuten unter anderem an die Vorschriften des Heilmittelwerbegesetzes halten. Diese geben zum Beispiel vor, dass sich Werbeaussagen nicht auf die Erkennung, Beseitigung oder Linderung von Krankheiten, Leiden, Körperschäden oder krankhaften Beschwerden beziehen dürfen. Für die Physiotherapie heißt das, dass die Therapeuten zwar für Behandlungsmethoden in der Praxis werben, jedoch keinen Erfolg versprechen dürfen. Die Möglichkeiten, seine Praxis zu bewerben, sind vielseitig. Doch bisher ist kaum etwas darüber bekannt, welche Werbemittel und -maßnahmen für physiotherapeutische Einrichtungen wirksam sind. Kerstin Weihs beschloss deshalb, diese Präferenzen in ihrer Bachelorarbeit näher zu untersuchen. Im Fokus stand dabei der Unterschied der Vorlieben von Patienten im ländlichen Raum und in der Stadt. In ihre Untersuchung bezog sie vier Physiotherapiepraxen ein, zwei ländlich (Landkreise Böblingen und Tübingen) und zwei städtisch (Stuttgart) gelegene. Dort befragte sie jeweils 50 Patienten per Fragebogen. Bei der Auswahl der Praxen achtete sie unter anderem darauf, dass dort bereits Werbemittel und -maßnahmen eingesetzt wurden und diese Einrichtungen etwa gleich viele Patienten innerhalb einer Woche behandelten. 184 Fragebogen konnte Kerstin Weihs auswerten. 55 Prozent der befragten Personen waren Frauen, und mit 41 Prozent kam die Mehrheit der Befragten aus demselben Ort, in dem sich die Praxis befindet. Fast die Hälfte (46 Prozent) der Befragten war zwischen 40 und 59 Jahre alt.

\section{Ergebnisse}

Kerstin Weihs hat herausgefunden, dass ...

> sich fast alle Patienten (91 Prozent) daran erinnern, wie sie auf die Praxis aufmerksam geworden sind.

$>$ für die Patienten aus der Stadt die Empfehlung des Arztes einen besonders hohen Stellenwert hat (50 Prozent), Patienten der ländlich gelegenen Praxen hingegen Wert auf eine Empfehlung von Freunden und Bekannten legen (64 Prozent).

> sich über das Internet vor allem Patienten aus der Stadt (15 Prozent) informieren. Bei denjenigen, die vom Land kommen, sind es nur zwei Prozent. Das Telefonbuch und Flyer werden von beiden Gruppen kaum als Informationsquelle genutzt. Dies liegt vor allem daran, dass Praxen bisher wenig mit Werbemitteln wie Flyern werben.

$>$ die Patienten, wenn man sie nach der Wichtigkeit einzelner Werbemittel fragt, vor allem die Praxis-Homepage (83 Prozent) und Flyer (70 Prozent) nennen.

\section{Fazit}

Zusammenfassend kann Kerstin Weihs festhalten, dass ...

$>$ die Mund-zu-Mund-Propaganda immer noch die wichtigste Werbung für eine Physiotherapiepraxis ist.

> es sich für eine Praxis auszahlen kann, in Werbemittel wie die Homepage oder Flyer zu investieren. Letztere können die Patienten zum Beispiel einfach an Freunde und Bekannte weitergeben.

$>$ umfangreichere Untersuchungen notwendig sind, um das Informationsverhalten von Patienten nachvollziehen zu können.

Eva Trompetter

$\Rightarrow$ Weihs, K. Werbung in der Physiotherapie. Ein Vergleich der Kundenreaktion auf Werbemittel von Praxen auf dem Land und in der Stadt. Bachelorarbeit an der Dresden International University; 2012. 